\title{
Enhancing Greedy Routing using Fuzzy logic for Vehicular Ad hoc Networks
}

\author{
Shilpy Agrawal, Ram Shringar Raw, and NeerajTyagi
}

\begin{abstract}
Intelligent Transportation Systems (ITS) has gain a lot of popularity due to much technological advancement in Vehicular Ad hoc Networks (VANETs). VANET provides safety and entertainment services to vehicle users. Therefore, constrained safety is a prominent feature for VANETs. Since the vehicular nodes typically moves fast than the nodes movement in other network such as Mobile Ad hoc Networks (MANETs), it is of great importance to design an efficient routing protocol to provide safety and comfort services to the passengers on the roads. Recently efforts have been put to use the concepts of fuzzy logic to help in the decision making process in VANETs. Fuzzy logic deals with reasoning that is approximate rather than fixed and exact. The fuzzy logic inference system became important and useful when the values of the decision criteria are not only vague but uncertain in nature. In this work, based on analysis of greedy routing for packet forwarding, we have proposed a Fuzzy Logic based Greedy Routing (FLGR) protocol. FLGR is a multihop routing protocol which is used to select the best next-hop node in multi-hop VANETs using fuzzy logic concept. We have considered two characteristics of a vehicle as an input metrics to fuzzy decision making systems. Based on the optimum function of simulation results, the FLGR effectively select the best next-hop node for further packet transmission in the network.
\end{abstract}

Key-Words: - Fuzzy logic, VANETs, routing protocol, greedy routing, FLGR, fuzzification.

\section{Introduction}

Wireless networks has created boom in today's era. These networks are being utilized in wide areas. They are being used for internet access, mobile communication, education, medical emergency care, travel etc. The major applications of this kind of network are safety and comfort applications for driver's driving the vehicles on roads, efficient traffic management applications, and infotainment services. A special class of networks known as Vehicular Ad hoc Networks (VANETs) [1] has been established by researchers which take care of above mentioned three applications. VANETs constitutes of large number of mobile nodes which are also known as vehicles whose movement is constrained to roads only.

Shilpy Agrawal, Neeraj Tyagi

Motilal Nehru National Institute of Technology, Allahabad, India

Ram Shringar Raw

Ambedkar Institute of Advanced Communication Technologies and Research, Delhi, India
Thus, compare to mobile ad hoc networks (MANETs) movement of nodes in VANETs are restricted to streets only and cannot move anywhere in any direction like the nodes in MANETs. The vehicles in vehicular networks are also known as smart vehicles as these vehicles are GPS (Global Positioning System) enabled and has wireless transceivers, central processing unit (CPU), and a wide variety of sensors embedded on it. In VANETs, communication take place either between vehicles or by a communication of a vehicle with a device deployed on roads known as road side units (RSUs). These RSUs serves as an access point and aids in efficient wireless communication. Thus, communication between vehicle-to-vehicle and between vehicle-to-road side units is known as V2V and V2I communication respectively. The two types of VANETs are shown in fig. 1.

In any of the above mentioned applications of VANETs, information dissemination from one vehicle to another is a critical task. Therefore, routing of data is one of the challenging tasks of vehicular networks. Safety and traffic monitoring related information is time-critical and has to be transmitted to the destination node/vehicle within a short time span. In cities the density of vehicles on roads is increasing day by day and it gets difficult to propagate the time-critical messages from source to destination node as these nodes can be very far away from each other. Therefore, there is a need of competent routing protocol that can efficiently transmit information from sender to receiver node. There are many types of routing protocols in VANETs like topology based, cluster based, broadcasting based, position based etc. but the most promising one is position based routing protocol.

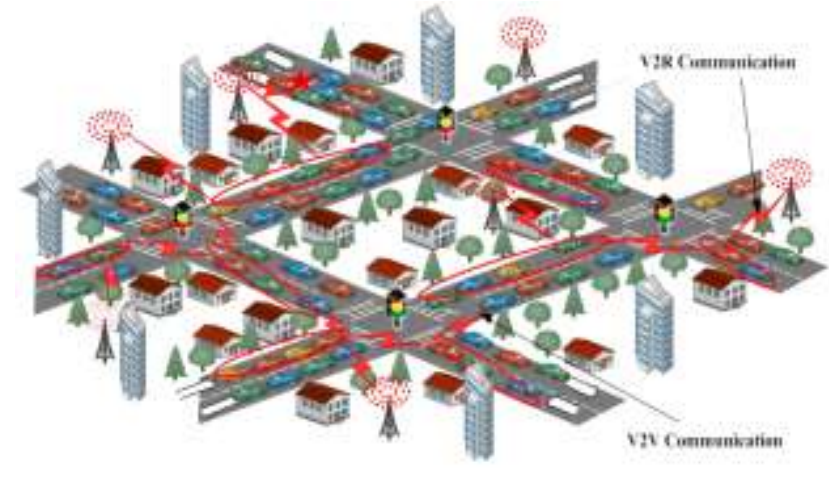

Figure 1. VANET Communications

In position based routing protocol the source node is aware of the location of its neighboring nodes and the position of destination node. The source node acquire these locations from On Board Units (OBUs) attached on vehicles or from street maps. The destination's information is obtained by source node from some location services [2]. Therefore, these 
Proc. of the Intl. Conf. on Advances in Computing, Control and Networking - ACCN 2015.

Copyright (C) Institute of Research Engineers and Doctors, USA .All rights reserved.

ISBN: 978-1-63248-038-5 doi: 10.15224/ 978-1-63248-038-5-81

position based routing protocols are very effective for highly dynamic networks like VANETs where vehicles move with a high speed leading to frequent topology changes.

In this work we have assumed that the source and destination node can be far away from each other on road and therefore we need many nodes between source and destination that helps in routing data packets. This type of routing where intermediate nodes are used for forwarding packets is known as multi hop routing. In this paper, we propose a greedy multi hop position based routing protocol that is based on the concepts of fuzzy logic [3][4]. The aim of the proposed work is to select the best next hop neighbor node while forwarding the information from source node to destination node in order to minimize the hop count and thus end-to-end delay. By doing so the time-critical safety messages in vehicular networks can reach timely within a short time span avoiding accidents, traffic jams etc.

We use the concept of fuzzy logic decision making system that helps in deciding which node is the approximate best next hop node for further forwarding the message in VANETs. The remainder of this paper is organized as follows. Section 2 gives a description of the proposed work and section 3 presents the results of simulations done using fuzzy tool box of MATLAB. Finally we present our conclusions in section 4.

\section{Fuzzy Logic Based Greedy Routing (FLGR) Protocol}

In this paper, we incorporate the concept of fuzzy logic system for selecting the best next-hop node for forwarding the packets and have proposed a fuzzy logic based greedy routing (FLGR) protocol. FLGR protocol helps in routing time-critical information with minimum end-to-end delay by deciding which neighbor node will be the approximate best next-hop node for further forwarding the packets and thus also minimizes the total hop count in a complete routing process from source to destination. Fuzzy logic is based on the concept of fuzzy set [5][6]. A fuzzy set is a set in which the membership of an object in a set is gradual rather than just member or not a member. The value of an object in fuzzy set lies in the range of real numbers between 0 and 1 . Unlike classical set where elements or members of a classical set will not be a member unless its membership is full, the elements of a fuzzy set have varying degree of membership in it. The entire fuzzy logic based system is divided into three steps, namely 1) Input (Fuzzification), 2) Process (Fuzzy Inference System), and 3) Output (Defuzzification).

Fuzzification [3] is the process of transforming a crisp (classical) set to a fuzzy set i.e. numerical values are converted to fuzzy values. This operation translates accurate numerical values into linguistic variables i.e. variables that can take words as its values in natural languages. These words are characterized by fuzzy sets defined in the Universe of Disclosure in which variable is defined [3]. The second step constitutes Fuzzy Inference System (FIS) that maps inputs by combining a set of membership functions with the control rules to fuzzy outputs. The two common types of FIS are Mamdani and Sugeno. In this work, we are using Mamdani fuzzy model. The last step is defuzzification. It involves the process of transposing the fuzzy outputs to crisp outputs. There are a wide variety of methods for defuzzifying the fuzzy output namely, centroid of area (COA), mean of maximum (MOM), fuzzy mean (FM) etc. Our work is focusing on COA method for Defuzzification.

The major motivation of the fuzzy logic system is to accomplish considerable improvement in the performance of the original greedy routing protocol through the improvement of selecting the next-hop forwarding node from a small subset of neighbor nodes for further transmission. In this paper, we use two metrics for selecting the best next-hop node, namely, distance metric which specifies the distance of a neighboring vehicle from source node/current forwarding node (CFN) and position metric which tells how far the neighboring vehicle is from the source/CFN on a straight line joining source/CFN and destination by projecting a projection on this straight line. The vehicles in VANETs are aware of the network information from the GPS device in the vehicle. A detail of these metrics is explained in the next section. It becomes difficult to decide the best next-hop node by using network information for the following reasons. First, the network information can be imprecise. Secondly, as these multiple metrics can conflict with each other, therefore it results in uncertainty. As fuzzy logic can deal with uncertain and imprecise information, we use a fuzzy logic based method to discover the neighbor node which can give the best results. The two metrics stated above serves as an input to fuzzy decision making system that helps in identifying the approximate best next-hop node for forwarding the packets.

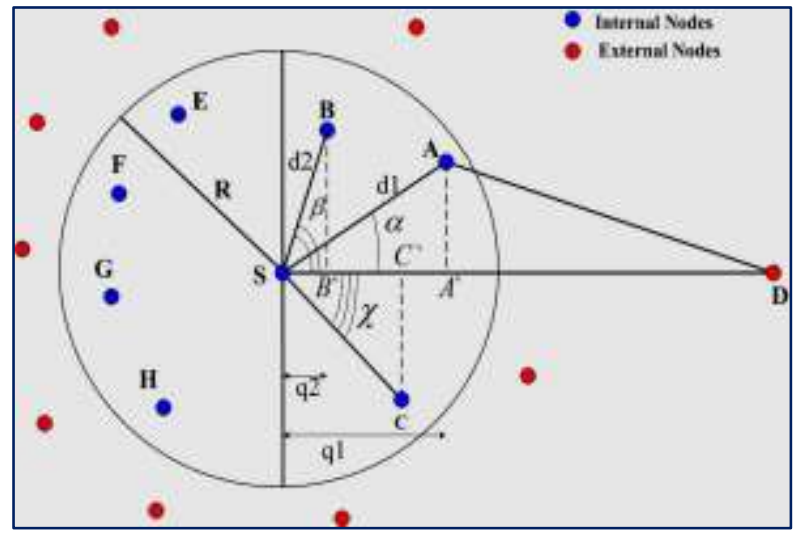

Figure 2. Selection of Node A as next-hop node in FLGR

The proposed protocol FLGR is used to select the next-hop forwarding nodes on the routing path through $\mathrm{V} 2 \mathrm{~V}$ communication processes. In FLGR, all the nodes in the network periodically broadcast the Hello packets. A sender node on receiving the Hello packet from the nodes in its transmission range $R$ gets aware of its neighbors. Next, the current forwarding node $(\mathrm{CFN})$ transmits a packet to a destination via intermediate nodes. Therefore, the CFN selects the best next-hop node among various other neighboring nodes from the right half of the circular region by employing the concept of fuzzy logic. During next-hop node selection process we consider two routing metrics such as distance of neighbor node from source, and position towards destination 
Proc. of the Intl. Conf. on Advances in Computing, Control and Networking - ACCN 2015.

Copyright $(\odot$ Institute of Research Engineers and Doctors, USA .All rights reserved.

ISBN: 978-1-63248-038-5 doi: 10.15224/ 978-1-63248-038-5-81

from source/CFN for each of the candidate neighbor nodes. These routing metrics are considered as an input of the fuzzy decision making system through which fuzzy output is calculated for each neighbors. The neighbor node with the maximum value of fuzzy output is selected as the best preferable next-hop neighbor node around a source/CFN. As shown in fig. 2, FLGR selects the node from the neighboring nodes as the next-hop node which is at maximum distance from the source node and closer to destination node.

$S:$ Source Node

CFN: Current Forwarding Node

TN: Total Number of Neighbors of CFN

SNN: Selected Next-hop Node

BNN : Best Next-hop Node

MAX: is a counter

Node id: Vehicle's Identification number

1. Set $\mathrm{CFN}=\mathrm{S}$

2. Define fuzzy sets for each input and output variables and represent them with membership functions for each input and output metric.

3. Generate fuzzy rules in the form of IF-THEN statements with the help of various routing metrics defined.

4. Set $\mathrm{MAX}=0$

5. If destination is in the maximum transmission range of $\mathrm{CFN}$, then exit.

6. for ( $i=1$, node_id=1; $i<=\mathrm{TN} ; \quad i++$, node_id ++

\{

7. Find classical data for the neighbor node i.e. distance from CFN, its progress towards destination.

8. Input this real world numerical (classical) data into fuzzy system.

9. Generate fuzzy values by using fuzzy MFs defined in step 2 for each input metrics.

10. Input the fuzzy values obtained in step 9 to fuzzy inference system.

11. Map the fuzzy values to pre-defined IF-THEN rules obtained in step 3 and combine all the rules together to obtain fuzzy output whose value reveals the best next-hop node.

12. Convert the linguistic result into a numerical value (defuzzify) using the predefined output $\mathrm{MF}$ and defuzzification method

13. if (crisp value $>=$ MAX)

\{

MAX = crisp value

SNN = node id;

\}

\}// end of for loop

14. Set $\mathrm{BNN}=\mathrm{SNN}$

15. Update $\mathrm{CFN}=\mathrm{BNN}$

16. Repeat steps 4 to 15.

17. End

Figure 3. Algorithm showing forwarding process in FLGR Protocol

This paper defines two types of nodes in the network, namely internal and external nodes. The neighbors that lie within the transmission range $R$ of the source/CFN i.e. inside the circular region as shown in the fig. 2 above are termed as internal nodes. Whereas the nodes outside the transmission range are known as external nodes. Fig. 2 shows that CFN is the source node $S$. Source node $\mathrm{S}$ has seven neighbors within its transmission range $R$, namely, $A, B, C, E, F, G$, and $H$. These neighbors are internal nodes. We discard $E, F, G$, and $H$ nodes as they are not in the direction of destination node and are far away from the destination too. Thus, we evaluate only the neighbors in the right half of the circular region as shown in the figure above. FLGR selects node $\mathrm{A}$ as the next-hop forwarding node as it positioned at maximum distance from source compare to nodes $B$ and $C(\mathrm{~d} 1>\mathrm{d} 2$ and $\mathrm{d} 1>\mathrm{SC})$ and has made maximum progress towards destination from current forwarding node as compare to other nodes. The entire proposed work is summarized in the algorithm given in fig. 3 .

\section{A. Performance Metrics Analysis}

- Distance: The distance metric indicates the distance of neighbor node from the source node within the transmission range $R$.

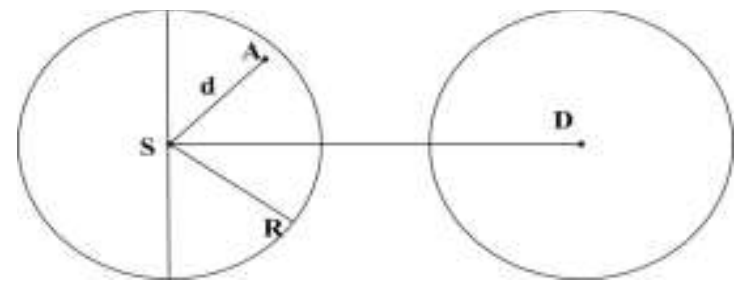

Figure 4. Distance Metric Calculation

The higher the value of distance, the more the node is closer to the destination node, thus following greedy method. Therefore, FLGR protocol forward traffic safety messages to next neighbors timely as the number of hops between source and destination gets reduced. A source/CFN upon receiving the Hello packets from its neighbor $A$, calculates the distance between itself and its neighbor $A$. We assume that all the vehicles are having same transmission range $R$ over which stable communications can take place. Therefore, the average distance between the two nodes will be [7]

$$
\operatorname{dist}(A)=\left\{\begin{array}{ll}
\frac{\mathrm{d}}{\mathrm{R}}, & \mathrm{d} \leq \mathrm{R} \\
1, & \mathrm{~d}>R
\end{array}\right\}
$$

Where, $d$ is distance between two nodes as shown in figure 4.

Position Metric: The current forwarding node is aware of the location of its neighboring nodes by the GPS device in the vehicle/node.

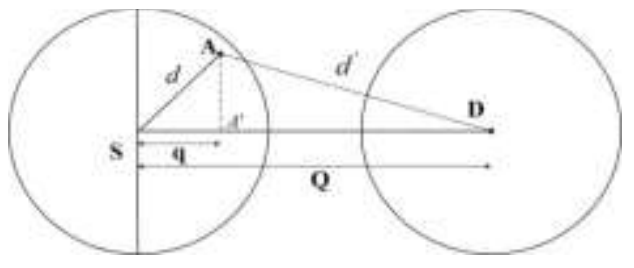

Figure 5. Position Metric Calculation 
Proc. of the Intl. Conf. on Advances in Computing, Control and Networking - ACCN 2015.

Copyright $(\odot$ Institute of Research Engineers and Doctors, USA .All rights reserved.

ISBN: 978-1-63248-038-5 doi: 10.15224/ 978-1-63248-038-5-81

Also the source node/CFN knows the position of destination node using some location service [2]. Therefore, it becomes easy to compute the position metric for any neighbor node in VANET. In [8], position metric $\left(\operatorname{Pos}_{\mathrm{NN}}\right)$ of a neighbor node has been defined as ratio of distance $(q)$ between forwarding node and a point $A^{\prime}$ on straight line $\mathrm{SD}$ as shown in the fig. 5. Projection $A$ ' of a neighbor node $A$ lies on the line SD having distances $Q$. Thus, position metric helps in finding the progress of neighbor node towards destination from source/CFN and helps in deciding the best next-hop node for further transmission. As shown in the fig. 5, the position metric is defined as given below

$$
\operatorname{Pos}_{\mathrm{NN}}=\frac{\mathrm{q}}{\mathrm{Q}}
$$

\section{Analysis of Results}

In order to develop the system to make routing easier, we take two important routing metrics, namely distance, and position stated above in the paper as input variables for fuzzification. We calculate an optimum fuzzy value using fuzzy toolbox of MATLAB [9] which decides the best nexthop forwarding node. We use Gaussian membership functions (MFs) in our analysis because it is suitable for highly dynamic random networks such as VANET. For each performance metrics, Gaussian membership function is used as defined in equation 3, based on which MFs for fuzzy sets are defined.

$$
\text { Gaussian }(x ; c, \sigma)=e^{-\frac{1}{2}\left(\frac{x-c}{\sigma}\right)^{2}}
$$

Where, $\mathrm{x}$ is a linguistic variable, $\mathrm{c}$ and $\sigma$ are MFs center and MFs width respectively. The distance metric is classified into five different linguistic variables, namely, very close, close, intermediate, far, and very far. The membership function of distance metric is defined in fig. 6 .

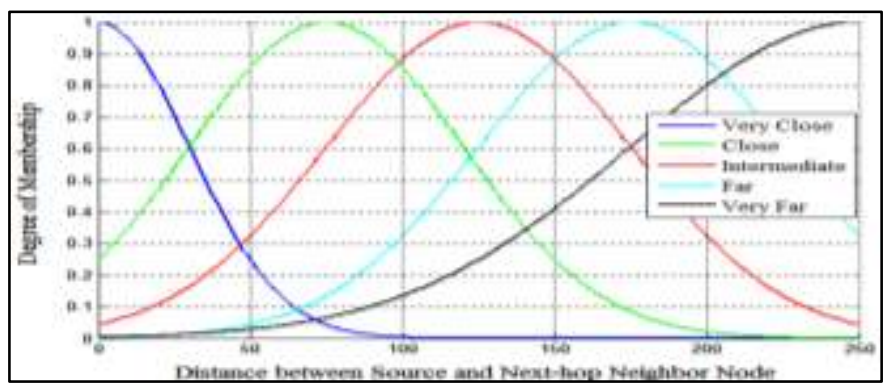

Figure 6. Distance Membership Function

The source/CFN uses these MFs of distance metric to compute which degree the distance factor belongs to i.e. very close, close, intermediate, far, and very far. Similarly, the position metric is classified into four membership functions according to the progress distance from the source vehicle and mathematically defined in fig. 7. Basically, source or CFN uses these membership functions of position metric to compute which degree the position metric belongs to \{very close, close, far, very far .

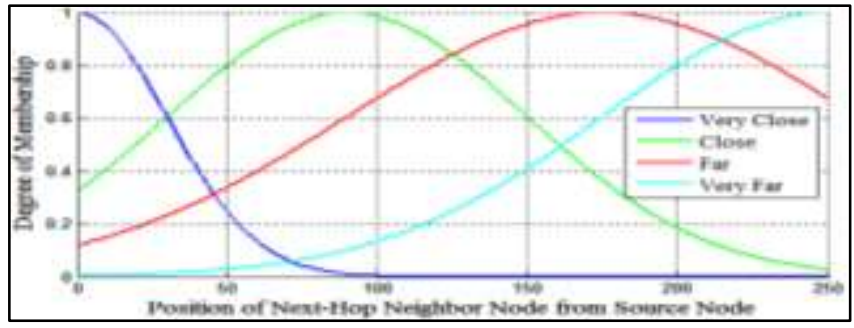

Figure 7. Position Membership Functions

So after fuzzification of the crisp data, we have designed a fuzzy inference engine that connects inputs and outputs based on the understanding of VANETs and routing metrics.

TABLE I. RULE BASE

\begin{tabular}{|c|c|c|c|}
\hline Rules & Distance & Position & $\begin{array}{c}\text { Optimum } \\
\text { Function }\end{array}$ \\
\hline Rule 1 & Very Far & Very Far & Very high \\
\hline Rule 2 & Very Far & Very close & Very Low \\
\hline Rule 3 & Far & Far & Very high \\
\hline Rule 4 & Far & Close & Low \\
\hline Rule 5 & Intermediate & Far & High \\
\hline Rule 6 & Intermediate & Close & Medium \\
\hline Rule 7 & Close & Close & Low \\
\hline Rule 8 & Close & Very Close & Very Low \\
\hline Rule 9 & Very Close & Very Close & Very Low \\
\hline
\end{tabular}

The fuzzy engine is designed based on 9 rules which are defined in table I. This fuzzy based IF-THEN rule is a conditional statement expressed as [3]

\section{IF < fuzzy preposition>, THEN<fuzzy preposition>}

Where, IF-part of the rule is known as antecedent and the THEN-part of the rule is known as the consequent. The sender node/CFN use IF-THEN rules (defined in table I) of fuzzy inference system to calculate the optimum function value of the current forwarding node. The optimum function value lies between 0 and 100, and is represented by linguistic variables defined as very low, low, medium, high, and very high. As multiple rules (table I) are applied at the same instance, so we have to combine their evaluation results. We use Min-Max method where for each rule; the minimal value of the antecedent is used as the final degree. When we combine different rules, the maximal value of the consequents is used. Consider Rule 1 of table I: IF Distance is Very Far, and Position is Very Far THEN Optimum Function is Very High.

When the distance of the neighbor vehicle is very large from the source/CFN within the communication range, then the number of nodes required for forwarding the time-critical safety messages can be reduced. Similarly, more the progress towards destination from $\mathrm{CFN}$ on a straight line joining CFN 
Proc. of the Intl. Conf. on Advances in Computing, Control and Networking - ACCN 2015.

Copyright $(\odot$ Institute of Research Engineers and Doctors, USA .All rights reserved.

ISBN: 978-1-63248-038-5 doi: 10.15224/ 978-1-63248-038-5-81

and destination, less number of hops will be there between source and destination.

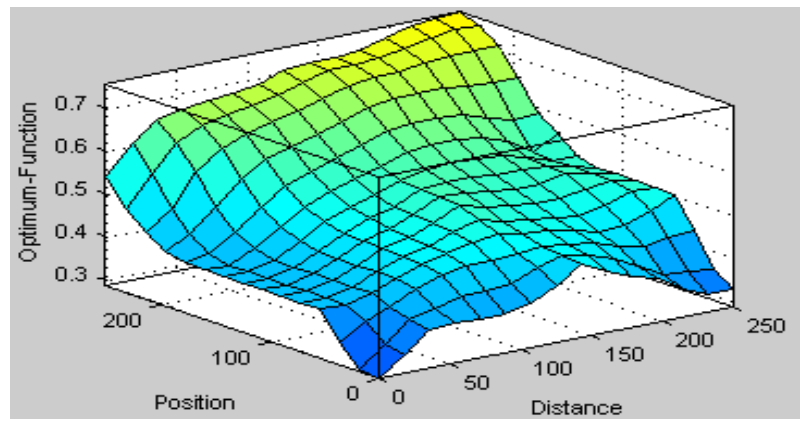

Figure 8. Optimum Function for various distances and position

Fig. 8 shows the correlation behavior between input and output variables. The 3-D graph in fig. 8 shows the value of optimum function which increases, when the distance of neighbor node from the source node/CFN is more than 190 meters and progress of neighbor node from CFN towards destination on a straight line joining CFN and destination increases (yellow portion of graph). In this way, keeping into mind all these facts and notions, we have designed rules to get the optimum value of the neighbor nodes. Among these neighboring nodes the node having maximum optimum function value will be selected as the next-hop forwarding node. After the processing step, we obtain the fuzzy result from the fuzzy inference system.

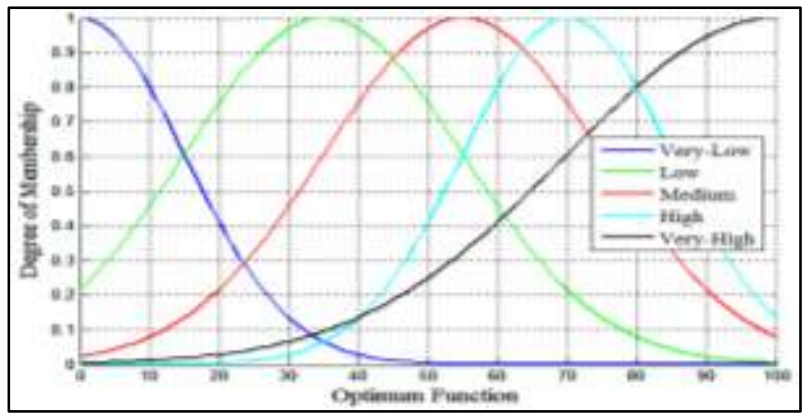

Figure 9. Output Membership Function

In the real world, we need a single numerical value as an output to any problem which can be used by any application instead of getting results in linguistic forms like very close, close etc. Therefore, in this work defuzzification has been applied to obtain the crisp value of optimum function based on output MF defined in fig. 9 and its corresponding membership degrees. There are many methods of defuzzification. Here we have used the centroid method which is the most widely used method and is calculated as

$$
\operatorname{COA}=\frac{\int \mu(x) x d x}{\int \mu(x) d x}
$$

Where, $x$ denotes fuzzy variable and $\mu(x)$ represents its membership function. It is also known as center of gravity or center of area (COA) method which gives the crisp value of the fuzzy optimum function. The higher the value of COA better will be the neighbor node for further forwarding the packets. Thus, in this way the propose protocol FLGR will be able to select that neighbor node as the best next-hop node which has maximum value of this optimum function and therefore helps in reducing the delay in delivering the packets in VANETs.

\section{Conclusions}

VANETs are based on wireless communication technology and are bringing a great revolution in the field of transportation systems. Vehicular networks harbor the promise of ubiquitous computing i.e. communication and mobile computing anytime, anywhere between vehicles-to-vehicles and vehicle-to-infrastructure. This paper focuses on the application of fuzzy logic systems in greedy multi-hop position based routing in VANETs. As fuzzy logic systems are an effective means of conflict resolution of multiple criteria and better assessment of options, therefore this system is used in this paper which helps in deciding the best possible nexthop neighbor node in routing using multiple metrics related to the network information. The simulation results indicate that when the distance of the candidate node is very far away from the packet carrier node, and its progress towards destination is more from CFN, then the fuzzy output function gives optimum result, and thus aids in the selection of that candidate node as a next-hop node.

\section{References}

[1] Fan Li., and $\mathrm{Yu}$ Wang, "Routing in vehicular ad hoc networks: A survey," IEEE Vehicular Technology Magazine, vol. 2, pp 12-22, June 2007.

[2] T. Camp, J. Boleng, L. Wilcox, "Location Information Services in Mobile Ad hoc Networks.", in Proc. IEEE International Conference on Communications(ICC), 2001, pp. 3318- 3324.

[3] G. J. Klier, B. Yan, Fuzzy Sets and Fuzzy Logic Theory and Applications. NJ: Prentice Hall, 1995.

[4] E. H. Mamdani, Application of fuzzy logic to approximate reasoning using linguistic synthesis, IEEE Transactions on Computers, vol. C-26, no.12, pp. 1182-1191, 1977.

[5] L. A. Zadeh, Fuzzy Sets, Info. \&Ctl. , vol. 8, 1965, pp. 338353.

[6] H. -J. Zimmermann, Fuzzy set theory and its applications, Kluwer, Boston, $2^{\text {nd }}$ edition.

[7] C. Wu, S. Ohzahata, T. Kato, Fuzzy Logic Based MultiHop Broadcast for High-Density Vehicular Ad Hoc Networks, IEEE Vehicular Networking Conference, pp. 17-24, 2010.

[8] R. S. Raw, D. K. Lobiyal, Improved Position-Based Routing in Vehicular Ad hoc Networks using P-DIR Method, in Advances in Computing and Communications (ACC), vol. 192, Springer Berlin Heidelberg, 2011, pp. 654- 663.

[9] www.mathsworks.in/help/fuzzy/foundations-of-fuzzylogic.html\#bp78170-7 